\title{
Study of RF Breakdown in Strong Magnetic Fields
}

\section{Alexey Kochemirovskiy*}

The University of Chicago

E-mail: kochemireuchicago.edu

Daniel Bowring, Katsuya Yonehara, Alfred Moretti

Fermi National Laboratory

\section{Yagmur Torun, Ben Freemire}

Illinois Institute of Technology

\begin{abstract}
RF cavities are essential components of almost every particle accelerator. Among other limitations, cavity gradients are restricted by occasional sparking inside the cavity, or RF breakdown. It has been shown that presence of external static magnetic field aggravates the influence of breakdown on performance of the cavity. This problem can arise in designs of muon ionization cooling channel, RF guns, klystrons and other applications. The MTA area at Fermilab is the facility that allows us to study the effects of static magnetic fields up to several Tesla on RF cavity operation. As a part of this research program, we have tested "modular" cavity in strong external magnetic fields. The special design of the cavity allowed for easier inspection of the cavity, swapping endplates with different materials and hence better control over sources of systematic errors. Results of high-power tests of "modular" cavity with and without external multi-Tesla magnetic field will be presented.
\end{abstract}

38th International Conference on High Energy Physics 3-10 August 2016

Chicago, USA

\footnotetext{
*Speaker.
} 


\section{Introduction}

\subsection{Motivation}

RF cavity is an essential component for any major accelerator system that allows to store the energy and transmit it towards beam acceleration. Typical structure of RF cavity is a metallic resonator kept in vacuum, connected to waveguide or coupler which enables power transmission to the cavity. Characteristic size of resonator varies between millimeter and meter scale while accelerating gradient is usually at least on the order of tens $\mathrm{MV} / \mathrm{m}$.

Among other limitations, cavity gradient is limited by RF breakdown: current discharge across a cavity accompanied by an abrupt drop in stored energy, bursts of light and x-ray emission, and a transient increase in vacuum pressure. Besides degrading the efficiency of beam acceleration, RF breakdown often damages surface structure of the cavity, which leads to an increase in probability of consecutive breakdown events.

Muon ionization cooling channel designs require RF cavities to be operated in strong solenoidal multi-tesla magnetic fields $[1,2]$. It has been experimentally demonstrated that presence of strong solenoidal magnetic fields increases the breakdown rates [3]. Beyond ionization cooling, this issue is relevant for any high-power RF system in external magnetic field, such as klystrons, photoinjectors and other applications.

\subsection{Mucool Testing Area (MTA)}

The MuCool Test Area (MTA) is a unique accelerator R\&D facility, built specifically for testing components for muon ionization cooling channel [4]. Cavities of 201 and $805 \mathrm{MHz}$ can be tested under high power with or without the presence of $400-\mathrm{MeV}$ proton beam supplied from Fermilab's Linac through special beamline. A superconducting solenoidal magnet with a maximum field strength of five Tesla is used to provide a magnetic field for both 201 and $805 \mathrm{MHz}$ cavity operations.

Automated RF cavity conditioning controls have been implemented in MuCool Test Area using LabView software [5]. Important features of this system include cavity waveform analysis during each pulse and breakdown detection; cavity tuning algorithm; gradient ramping and recovery from breakdown; capability for saving regular waveforms, triggered breakdown waveforms and text $\log$ files to disk for future data analysis.

\section{Problem of RF Breakdown in Strong Magnetic Field}

A model explaining the effect of strong magnetic fields on RF breakdown rates has been proposed in [6]. In this model, in the presence of external magnetic field, electrons emitted by microscopic surface defects are focused into "beamlets" that persist over multiple RF cycles and, upon impacting on cavity surfaces, lead to material degradation through cyclic fatigue. Eventually, local surface damage is formed and may trigger RF breakdown event. For magnetic field exceding some threshold value, focusing effects get counteracted by space charge effect and leads to diminishing increase in beamlet current density. The model assumes that mechanical stress induced on the surface of the metal by local temperature rise grows large enough to cause substantial damage 
when $\Delta \mathrm{T}$ exceeds the safe temperature of copper. As a consequence of the model, there are several factors that may decrease the breakdown rate:

- Surface preparation techniques that reduces the number of surface defects causing electric field enhancement

- Usage of materials with higher radiation length, such as Beryllium, that allow electrons to pass through without deposing energy in the cavity walls

- Geometrical solutions that decrease the impact energy density of electrons

Number of $805 \mathrm{MHz}$ pillbox-like cavities have been tested in MTA at high power in external magnetic field up to 5 Tesla [7]. Their gradient performance is shown in Figure 1. In general, gradient behavior with external magnetic field follows prediction, however, there are some factors that may affect the fit quality. The relatively poor gradient performance of cavity represented by yellow points on the graph can be explained by lack of surface treatment received by cavity walls. So-called "All-Seasons Cavity" (blue trace on the graph) had a longer body length that resulted in decreased impact energy of electrons traversing the accelerating gap of the cavity. The concept of cavity with gridded windows (red and green traces) envisaged lowering the density of material in the path of emitted electrons. For all cavities featured on this graph, designs of power transmission systems to the cavity resulted in causing the electric field enhancement region around coupler and consequently an increase in breakdown probability in that region. This factor, therefore, inadvertently created an additional source of systematic error for studying the problem of RF breakdown.



Figure 1: Peak surface electric field $v s$. external magnetic field for various $805 \mathrm{MHz}$ pillbox-like cavities. The black line corresponds to prediction of the model discussed above. More detailed discuss of test results can be found in [7].

\section{Modular Cavity Program}

New cavity was designed and built with the goal of better experimental flexibility and improved control over sources of systematic error [8]. The flat walls of the cavity can relatively easy 


\begin{tabular}{ccc} 
Date & Magnetic field & Peak Gradient \\
\hline October'15 & $0 \mathrm{~T}$ & $45 \mathrm{MV} / \mathrm{m}$ \\
January'16 & $3 \mathrm{~T}$ & $12 \mathrm{MV} / \mathrm{m}$ \\
February'16 & $0 \mathrm{~T}$ & $22 \mathrm{MV} / \mathrm{m}$ \\
April'16 & $3 \mathrm{~T}$ & $10 \mathrm{MV} / \mathrm{m}$ \\
\hline
\end{tabular}

Table 1: Summary of high-power tests of Modular Cavity. "Peak gradient" is the maximum surface gradient reached within the allowed breakdown rate of 1 spark in $10^{5} \mathrm{RF}$ pulses.

be removed for inspection or reconditioning of inner surfaces and allow for materials swap, hence the structure is referred to as the "modular cavity". The cavity walls were chemically polished and special design of input coupler provides a factor of $>5$ suppression of electric field around coupler region compared to on-axis electric field of TM010 mode.

\subsection{Experimental Results}

Initial high-power run of modular cavity was performed in zero external magnetic field with fresh set of copper endplates to establish the baseline gradient performance of the cavity. The maximum gradient of $45 \mathrm{MV} / \mathrm{m}$ was established and the flat endplates were removed for surface inspection. Figure 2 demonstrates the typical damage observed by microscopic inspection. It is not clear if the damage observed is a direct result of breakdown events. Other potential causes might be field emission or process of removal of subsurface impurities during high-power conditioning. Important to note that the damage is microscopic and, within the measurement precision of 5 micrometers, does not alter the roughness of the surface.
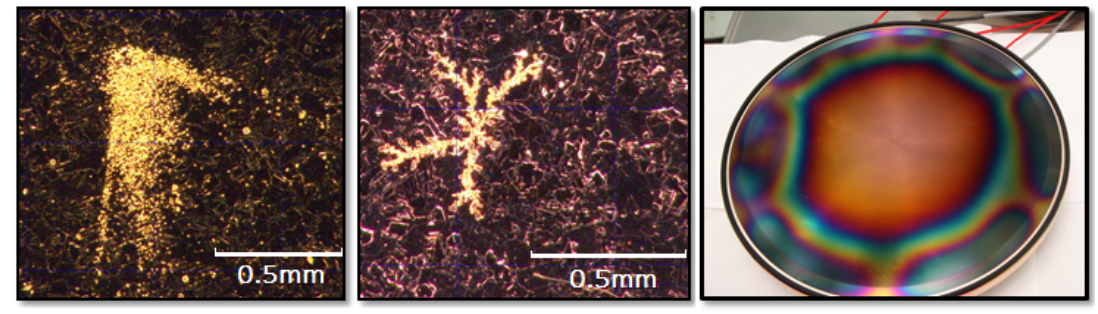

Figure 2: Typical surface damage observed after high-power $\mathrm{B}=0 \mathrm{~T}$ run and interior surface of flat cavity wall. The variation in surface coloring on the endplate is due to non-uniformity of TiN coating thickness.

After establishing the baseline performance in zero external magnetic field, the cavity was tested at $3 \mathrm{~T}$. As we observed rate of breakdown exceeding target rate of $10^{-5}$ sparks per pulse while operating at gradients below $12 \mathrm{MV} / \mathrm{m}$, the run was interrupted for surface inspection after detecting 55 breakdown events.

168 new damage sites were observed on each flat cavity wall. Digital micrographs of typical breakdown damage sites are shown in Fig. 3. All these new pits are round shaped with diameter between 1 and $2 \mathrm{~mm}$. Some of the pits have a core of burnished, apparently melted and solidified copper at their centers.

The cavity walls were scanned at 1800 dpi resolution and the coordinates of the breakdown sites were recorded. The endplates show a near-perfect mirror image of damage accumulated 

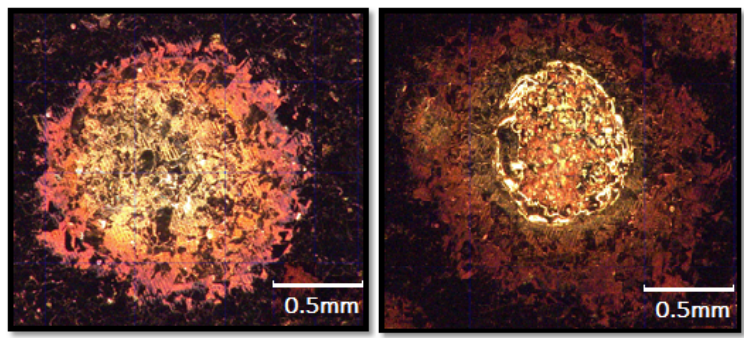

Figure 3: Microscopic images of typical breakdown "pits" with and without melted core present.



Figure 4: Map of breakdown damage from a reference point of downstream endplate, upstream coordinate are flipped in X. Red and blue dots correspond to pits on the opposing flat walls.

during this run, as shown in Fig. 4. As surface inspections were performed after each run, we can state that all the "pits" were inflicted during operation at $B=3 \mathrm{~T}$.
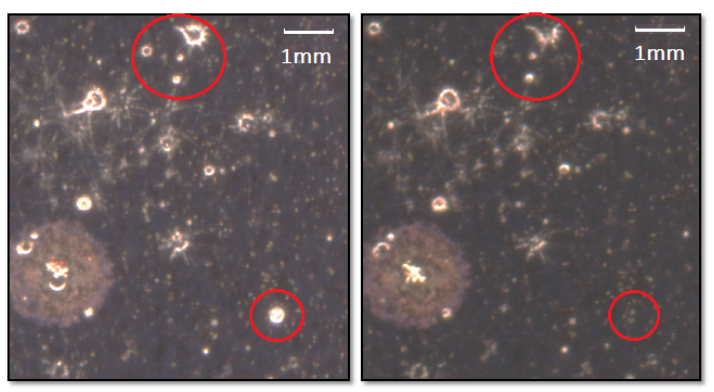

Figure 5: Copper "splashes" observed after B=3 $\mathrm{T}$ run (left) and the same region (right) after the subsequent $\mathrm{B}=0 \mathrm{~T}$ conditioning run. Disappearance of spots is highlighted.

Inspection after the $\mathrm{B}=3 \mathrm{~T}$ run showed significant damage on the surface of endplates. It is likely that surface damage from previous runs caused breakdown to occur at gradients lower than the first $B=0$ run. Previous experience operating similar cavities indicates that conditioning in $0 \mathrm{~T}$ may help to process through defects on the surface created during high field operation and subsequently improve the performance in $3 \mathrm{~T}$ [9]. The cavity was conditioned up to $22 \mathrm{MV} / \mathrm{m}$ 
after running for 7 million RF pulses and detecting approximately 450 sparks. After this run, another inspection was performed. No new damage traces were observed. Some copper spots (likely solidified splashes of liquid metal) seems to have disappeared, as shown in Fig. 5. After conditioning run, cavity was again tested in $\mathrm{B}=3 \mathrm{~T}$ field. Safe operating gradient was established to be $10.2 \pm 0.5 \mathrm{MV} / \mathrm{m}$.

\section{Conclusion}

Number of pillbox cavities were tested in MTA in strong external fields up to 5 T. Gradient behavior of these cavities agrees with the introduced model RF breakdown in strong magnetic fields. To improve experimental flexibility and control over sources of systematic error, new cavity with removable flat end walls was designed, built and tested at high power. Several inspections were performed after each run to track the correlation between damage forming processes and run conditions. Observed variance in damage characteristics between $\mathrm{B}=0 \mathrm{~T}$ and $\mathrm{B}=3 \mathrm{~T}$ runs indicates different energy deposition mechanisms: presence of strong external magnetic field produces more "violent" damage that prevents stable operation at high gradients. Observed perfect one-to-one correspondence between the pits on the opposing flat endplates after 3 tesla run supports the model of breakdown being induced by focused dark current beamlets inside the cavity.

The next steps in Modular Cavity experimental program will involve operation with Beryllium endplates instead of copper. We will test the hypothesis that Beryllium, material with smaller radiation length than copper, will ameliorate the gradient performance of the cavity in strong magnetic field. Additionally, dark current electrons passing through thin (1mm) 1-inch-diameter Beryllium window will enable us to directly measure field emission current and track surface evolution.

\section{References}

[1] R. Palmer et al., "Muon Collider Design", AIP Conf. Proc. 441 (1998) 183.

[2] A. Blondel et al., "MICE, The International Muon Ionization Cooling Experiment", TUPFI046, IPAC'13.

[3] A. Moretti et al., "Effects of high solenoidal magnetic fields on rf accelerating cavities", Phys.Rev. ST Accel.Beams 10 (2005) 072001.

[4] http://www.mice.iit.edu/mta/

[5] D. Petersen and Y. Torun, "Fermilab Mucool Test Area cavity conditioning control using LabView", Fermilab-conf-13-167-AD.

[6] D. Stratakis et al., "Effects of external magnetic fields on the operation of high-gradient accelerating structures”, Nucl. Inst. Meth. A 620 (2010), 147-154.

[7] D. Bowring et al., "RF Breakdown of 805 MHz Cavities in Strong Magnetic Fields", MOAD2, IPAC'15.

[8] D. Bowring et al., "RF Design and Operation of a Modular Cavity For MuonIonization Cooling R\&d", MOOCA02, IPAC'14.

[9] J. Norem, V. Wu, A. Moretti, M. Popovic, Z. Qian, L.Ducas, Y. Torun, and N. Solomey, Phys. Rev. ST Accel. Beams 6, 072001 (2003). 